\title{
Left atrial function and NT-proANP as markers of AF progression and impaired outcome in patients with heart failure with preserved ejection fraction
}

\author{
Timm Seewöster $^{1}$ (D) Jelena Kornej ${ }^{2}$
}

Received: 4 September 2019 / Accepted: 16 September 2019 / Published online: 5 October 2019

(C) Springer Nature B.V. 2019

Heart failure (HF) is a frequent condition associated with diverse cardiac comorbidities such as cardiac arrhythmias. One of the common underlying diseases in preserved ejection fraction (HFpEF) and atrial fibrillation (AF) is hypertension. Hypertension leads to left ventricular wall thickening and impaired ventricular relaxation, which adds up to left atrial (LA) dilatation contributing to electro-anatomical remodeling. Diabetes and female gender are also important risk factors in both HFpEF and AF. For example, female gender is associated with the presence of HFpEF rather than with heart failure with reduced ejection fraction, which is more common in men. Also, females have a higher risk for the presence of low voltage areas (LVAs) compared to men. Diabetes is an indicator not only for worse outcome of HFpEF patients, it is also a variable included into LVAs prediction score.

LA size enlargement is associated with AF initiation and progression. In patients with HFpEF LA dilatation leads to the development of pro-arrhythmic substrate based on atrial fibrosis. Electro-anatomical remodeling is an expression of fibrotic changes in LA and could be measured with electroanatomical mapping during invasive AF treatment as LVAs. Atrial natriuretic peptide (ANP), the hormone produced by the atria in response to stretch resulting in atrial fibrosis, might be considered as a link between HFpEF and AF.

In the current study Kanagala and colleagues analyzed the prognostic impact on reduced left atrial ejection fraction

This comment refers to the article available online at https://doi. org/10.1007/s10554-019-01684-9

Timm Seewöster

t.seewoester@yahoo.de

1 Department of Electrophysiology, Heart Center, Leipzig, Germany

2 School of Medicine - Cardiovascular Medicine, Boston University, Boston, USA
(LA-EF) on clinical outcomes as re-hospitalization because of heart failure or death. The authors found that decreased LA-EF measured in cardiovascular magnetic resonance imaging (CMR) remained a significant predictor for the combined endpoint. Furthermore, the authors showed that elevated NT-proANP levels significantly predicted re-hospitalization and death.

We'd like to congratulate the authors presenting these interesting results. Although the main focus in this study was the role of LA-EF in HFpEF patients, we would like to highlight the LA-EF importance in patients with AF, as both conditions-HFpEF and AF-frequently coexist.

While persistent AF type and increased left atrial diameter are well-established indicators for AF progression, another important variable associated with AF progression and severity stage is periprocedural evidence of LVAs representing advanced remodeling processes in the left atrium. Recently we demonstrated that CMR-derived LA-EF is associated with $\mathrm{AF}$ progression phenotypes and the presence of LVAs. Although there was a significant association between NT-proANP and different LA size parameters, such as LA volume, anterior- posterior, superior- inferior and transversal diameter, the LA volume remained most significantly associated with biomarkers [1]. In another study, however, the association between advanced AF progression and LA-EF was stronger than with LA size only [2] .

Summarizing, LA function and NT-proANP might be considered as biomarkers linking AF progression and HFpEF patients, indicating a poorer outcome and advanced disease stages. These biomarkers could be helpful to improve patients' selection for different therapeutic strategies and individualize AF and HF management.

Author contributions All authors contributed to the conception and design of the manuscript. The first draft of the manuscript was written 
by TS and JK commented on previous versions of the manuscript. All authors read and approved the final manuscript.

\section{Compliance with ethical standards}

Conflict of interest The authors declare that they have no conflict of interest.

Ethical approval In all studies, which are cited in the manuscript, data were collected in accordance with the Declaration of Helsinki, after approval of the local institutional review board.

Informed consent All patients provided written informed consent.

\section{References}

1. Seewöster T, Büttner P, Nedios S, Sommer P, Dagres N, Schumacher K, Bollmann A, Hilbert S, Jahnke C, Paetsch I, Hindricks
G, Kornej J (2018) Association between cardiovascular magnetic resonance-derived left atrial dimensions, electroanatomical substrate and NT-proANP levels in atrial fibrillation. J Am Heart Assoc 7(19):e009427

2. Seewöster T, Spampinato RA, Sommer P, Lindemann F, Jahnke C, Paetsch I, Hindricks G, Kornej J ( 2019) Left atrial size and total atrial emptying fraction in atrial fibrillation progression. Heart Rhythm. https://doi.org/10.1016/j.hrthm.2019.06.014

Publisher's Note Springer Nature remains neutral with regard to jurisdictional claims in published maps and institutional affiliations. 